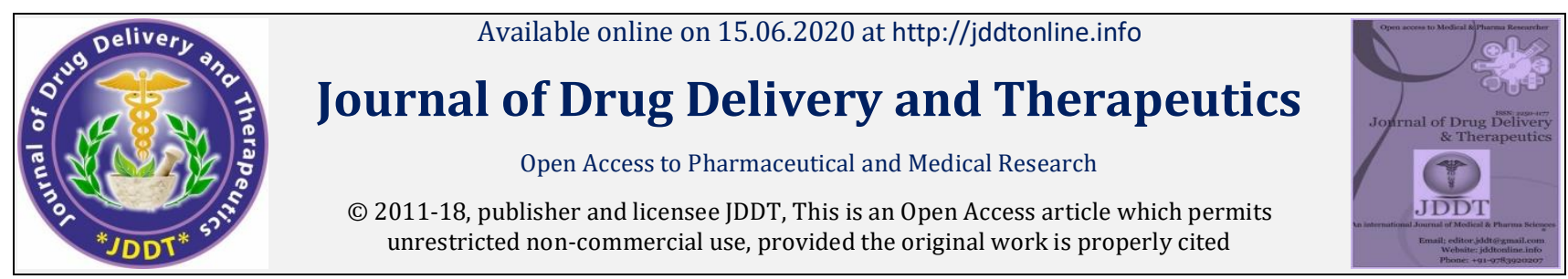

Open Access

Research Article

\title{
A Study on Medication Adherence and Quality of Life in Patients with Chronic Kidney Disease
}

\author{
Christeena S Varghese ${ }^{1}$, Harshita Krishna Naik ${ }^{1}$, R Sangeetha' ${ }^{1}$, R Srinivasan*2 \\ 1 Pharm D Interns, Department of Pharmacy Practice, PES University, Bengaluru, Karnataka, India-560 050 \\ ${ }^{2}$ Chairperson, Department of Pharmacy Practice, Faculty of Pharmaceutical Sciences, PES University, Bengaluru, Karnataka, India-560 050
}

\begin{abstract}
Back ground: Chronic Kidney Disease (CKD) is the most common health related problem. Poor long term adherence is the major clinical issue in the management of CKD. Therefore good medication adherence is important to obtain desired therapeutic outcome which in turn improves the quality of life of the patient. The aim of the study is to carry out a study on medication adherence and to improve health related quality of life in patients with CKD.
\end{abstract}

Objectives: To evaluate medication adherence and to assess the factors associated with non adherence and to provide patient counselling to improve medication adherence and to improve their quality of life.

Methods: A prospective study conducted for a period of six months in NU Hospitals Bengaluru. A total of 140 patients (62 were undergoing hemodialysis, 66 were CKD stage 3-5 and 12 were post-transplant patients) were enrolled for the study. Patient's adherence to medication was assessed with the help of Modified Morisky, Green, Levine Medication Adherence Scale and Quality of life was assessed with the help of Kidney Disease Quality of Life-Short Form 36 questionnaire. One to one follow up was done after 3 months of initial data collection.

Results: During the study 140 patients were enrolled for the study with 103 males and 37 females. Patient with low and medium adherence during the first visit were 7 (5\%) and 21 (45\%) respectively and patients with high adherence during baseline visit were $80 \%$, which later improved to $85.7 \%$ in the final review after patient counselling. Quality of life is decreased in patients with CKD. The domain with highest average score was emotional well being (8.4) and lowest average score was role limitation due to physical health (4.12). There was a slight improvement in Quality of Life of the patients after patient counselling.

Conclusion: Study suggests that patient counselling by clinical pharmacists can improve the medication adherence by improving awareness about medication which in turn helps them to achieve a better quality of life.

Keywords: Chronic Kidney Disease (CKD), medication adherence, quality of life, patient counselling.

Article Info: Received 28 March 2020; Review Completed 23 May 2020; Accepted 28 May 2020; Available online 15 June 2020

Cite this article as:

Varghese CS, Naik HK, Sangeetha R, Srinivasan R, A Study on Medication Adherence and Quality of Life in Patients with Chronic Kidney Disease, Journal of Drug Delivery and Therapeutics. 2020; 10(3-s):52-60

http://dx.doi.org/10.22270/jddt.v10i3-s.4083

D.

Prof. (Dr.) R. Srinivasan, Chairperson, Department of Pharmacy Practice, Faculty of Pharmaceutical Sciences, PES University, Hanumanth nagar, Bengaluru, Karnataka, India-560 050.

\section{INTRODUCTION}

Chronic nephropathy (CKD) conjointly referred to as chronic nephrosis or progressive nephropathy or uropathy. It is defined as the presence of excretory organ harm or ablated capillary Filtration Rate (GFR). CKD could be a progressive un-wellness that eventually ends up in nephropathy known as End-Stage excretory organ diseases (ESRD). Therefore early detection, management and treatment is considered in minimizing morbidity and mortality ${ }^{1}$. The incidence of CKD has doubled in last 15years. CKD has become a pestilent as a result of sophisticated interrelationships with DM, high blood pressure and different associated diseases ${ }^{2}$.

CKD patients belongs to the subjects with daily pill load up to twenty pills per day relying upon the severity of the disease; such a high pill burden is inevitably related to major issues of drug adherence ${ }^{3}$.

\section{Adherence to medication:}

Adherence to medication is the extent to that the patient's behaviour matches with the recommendations from the prescriber ${ }^{4}$. World Health Organization (WHO) data shows 
that only $50 \%$ of the individuals with chronic illness take their medication properly as suggested. Thus non adherence to prescribed medication is related to poor clinical outcomes, accelerated illness progression and big economic burden to health care system. Therefore, assessment of adherence to prescribed treatment plan is very important in reducing the complexities of chronic illness. Poor compliance is the most typical explanation for failure to retort to medications and poor treatment outcomes. Poor compliance typically ends up in further unessential tests, indefinite quantity changes, emergency department visits or medical aid that ultimately leads to inflated cost of medical care. In CKD adherence to medication could be a key element for effective management. Poor adherence and persistence to drug is universally recognized as the foremost clinical problems within the management of chronic diseases. Non adherence rates among CKD patients found in previous studies vary from two to ninety eight percent, hence it's necessary to know non adherence to and study the factors associated. Many studies showed that patient medical education will retard the progression of chronic diseases and improve survival, improves sickness understanding and boost quality of life ${ }^{4}$.

\section{Potential methods to enhance medication adherence in CKD patients:}

Strategies to enhance drug adherence in CKD patients need to primarily concentrate on the patient and physician's motivations as well as developing trust and sympathy providing the maximum patient education on the requirement of taking all of those medication. While evaluating drug therapies, the main focus should be simplifying drug regimens ${ }^{4}$.

\section{Quality of life (QOL) in CKD patients:}

World Health Organization (WHO) defines quality of life (QOL) as an individual's perception of their position in life within the context of the culture they live and in relation to their goals, standards and concerns. It is affected by the person's physical health condition, personal belief, social relationship and their relationship to the environment.Most conceptualizations of QOL emphasize the results of illness on physical, social/role, psychological/emotional, and psychological feature functioning. Symptoms, health perceptions, and overall quality of life are typically enclosed within the domain of QOL 5 .

Effective management depends on educating the patient about the importance of taking medications which in turn improves their medication adherence and thus decreases the progression of the disease. By counselling a patient can help in achieving increased medication knowledge, decreased hospitalization rates and thus improves the overall quality of life of the patients 5 .

\section{Objectives}

$>$ To evaluate the adherence to medication and to assess the factors associated with non-adherence.

$>$ To provide patient counselling to improve medication adherence and to improve their quality of life.

\section{MATERIALS AND METHODS}

\section{Study site:}

The study was conducted at NU Hospitals, Padmanabha nagar, Bengaluru, Karnataka, India.

\section{Study design:}

A prospective observational study.

\section{Study duration:}

This study was carried out for a period of six months.

\section{Inclusion criteria}

- Patients who are attending hospitals, Nephrology outpatient's department and patients undergoing hemodialysis irrespective of co-morbidities.

- Patients who are above 18 years of age.

- CKD patients of stage 3-5D (D-dialysis).

- Renal transplant patients.

\section{Exclusion criteria}

- Patients who are not willing to give the Informed consent.

- $\quad$ Patients less than 18 years of age.

- $\quad$ CKD stages 1-2.

\section{Source of data collection:}

- The data necessary for the study was collected from,

○ Patients case files,

- Interviewing patients and their care takers.

\section{Informed consent procedure:}

The study plan was explained in detail to the patient with the help of Patient Information Sheet. Informed Consent is taken in a format approved by Ethics Committee, NU Hospitals in either English/Kannada. Consent form was explained to the patient by the investigator before obtaining the signature.

\section{Study procedure:}

After obtaining the Informed consent from the patient, information such as demographic details (after hiding the patient's identity), co-morbid factors (hypertension, diabetes mellitus, anaemia, cardiovascular disorders etc.), medication taken and the frequency of dialysis was collected with the help of patient data collection form. Patients GFR were calculated using Modification of Diet in Renal Disease (MDRD) equation.

One to one follow up was done after 3 months of initial data collection. Patients adherence to medication was assessed with the help of Modified Morisky, Green, and Levine Medication Adherence Scale approved by Ethics Committee, NU Hospitals. The questionnaire contains 8 questions that identify the reasons for non-adherence such as:

\section{- Forgetfulness}

- Carelessness

- Lack of medication information

- Fear of side effects

- Pill burden

- Cost of drug

Scores are given according to the answers given by the patient. For each question, there are 2 possible answers, YES or NO. Adherence score for these 2 answers were set as $\mathrm{NO}=0$ and $\mathrm{YES}=1$ except for 7 th question were $\mathrm{NO}=1$ and YES $=0$ and for $8^{\text {th }}$ question there are 4 possible answers like a) never, b) once in a while, c) sometimes, d) usually, e) all the time where $\mathrm{a}$ is scored as 0 and $\mathrm{b}$ to e is scored as 1 . 
Adherence was calculated based on the total score of the medication adherence questionnaire. Scores, $>2$ indicates low adherence, score 1-2 indicates medium adherence and score 0 indicates high adherence. The patient's medication adherence decreases as the score of the medication adherence questionnaire increases. Quality of life was assessed with the help of KDQOL-SF-36. The questionnaire contains nine health concepts such as:

- Physical functioning

- Role limitation due to physical health

- Role limitation due to emotional problems

- Energy/fatigue

- Emotional well being

- Social functioning

- Pain

- General health and

- Health change

Scoring was done using online RAND 36 Score calculator. It is a two-step process. First, preceded numeric values are recoded, high score defines a more favourable health state. In addition, each item is scored on a 0 to 10 range so that the lowest and highest possible scores are 0 and 10 respectively. Scores represent the percentage of total possible score achieved. In step 2, items in the same scale are averaged together to create the 9 scale scores.

\section{Aid used:}

Patient Information Leaflet was taken from American Academy of Family Physicians which was given to the patients after patient counselling to improve their quality of life. It gives out information about the importance of taking medicines, lifestyle modification such as exercises and diet.

\section{Data collection form:}

The data collection form was developed. It includes patient demographics, comorbidities, number of medicines taken, other medicines taken (OTC, Ayurvedic etc), serum creatinine and stages of CKD.

\section{Statistical analysis:}

$\checkmark$ Correlation between medication adherence and quality of life was assessed with the help of Pearson Correlation.

$\checkmark$ Medication adherence behaviour before and after patient education was compared with the help of paired sample T-test.

\section{RESULTS}

A total of 140 patients (62 were undergoing hemodialysis, 66 were CKD stage $3-5$ and 12 were CKD transplant patients) were enrolled for the study, out of which 103 were males and 37 were females.
Table 1: Gender wise distribution of patients

\begin{tabular}{|ccc|}
\hline Gender & Frequency & Percent (\%) \\
\hline Male & 103 & 73.57 \\
\hline Female & 37 & 26.42 \\
\hline Total & 140 & 100.0 \\
\hline
\end{tabular}

Table 2: Patient distribution according to age

\begin{tabular}{cc}
\hline Age & Total number of patients (\%) \\
\hline $20-29$ & $7(5)$ \\
$30-39$ & $11(7.86)$ \\
$40-49$ & $13(9.3)$ \\
$50-59$ & $34(24.28)$ \\
$60-69$ & $46(32.9)$ \\
$70-79$ & $25(17.85)$ \\
$80-89$ & $4(2.85)$ \\
\hline
\end{tabular}

Table 3: Patient distribution according to different stages of CKD

\begin{tabular}{|ccc|}
\hline Stage & Number of patients & Percent (\%) \\
\hline 3A & 6 & 4.28 \\
\hline 3B & 16 & 11.42 \\
\hline 4 & 25 & 17.85 \\
\hline 5 & 19 & 13.57 \\
\hline $5 \mathrm{D}$ & 62 & 44.28 \\
\hline $\begin{array}{c}\text { CKD } \\
\text { Transplant }\end{array}$ & 12 & 8.57 \\
\hline Total & 140 & 100 \\
\hline
\end{tabular}

Table 4: CKD with other co-morbidities

\begin{tabular}{|c|c|}
\hline Comorbity & Number of patients (\%) \\
\hline HTN & $132(94.2)$ \\
\hline DM & $86(6104)$ \\
\hline Anemia & $52(37.1)$ \\
\hline CV Disorder & $28(20)$ \\
\hline Thyroid & $21(15)$ \\
\hline Respiratory disorder & $10(7.14)$ \\
\hline
\end{tabular}



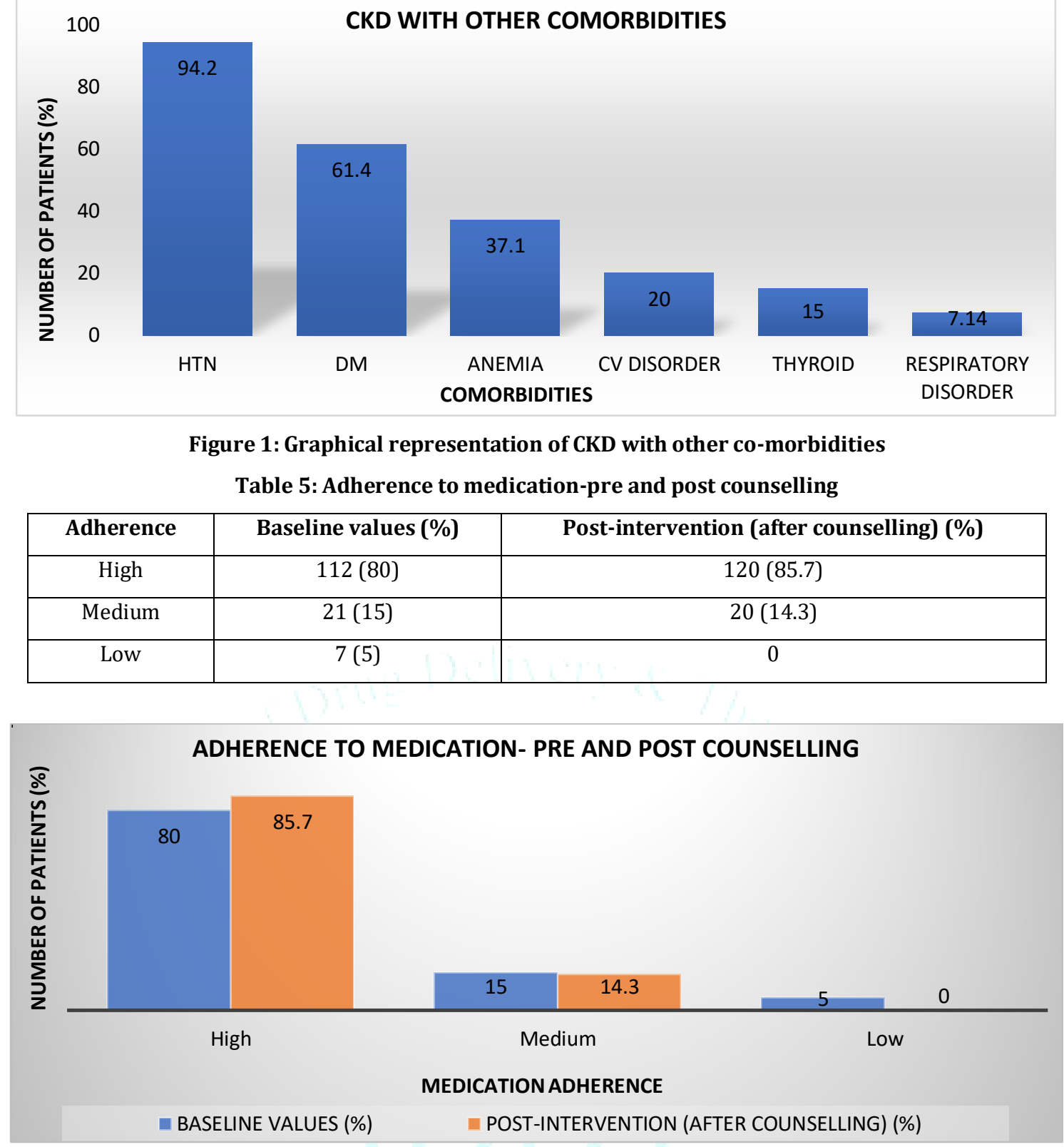

Figure 2: Graphical representation of Adherence to medication-pre and post counseling

Table 6: Medication adherence-pre and post counselling among male patients

\begin{tabular}{|c|c|c|}
\hline Adherence & Baseline value (\%) & Post-intervention (after patient counselling) (\%) \\
\hline High & $82(79.61)$ & $89(86.40)$ \\
\hline Medium & $15(14.5)$ & $14(13.59)$ \\
\hline Low & $6(5.82)$ & 0 \\
\hline
\end{tabular}

Table 7: Medication adherence-pre and post counselling among female patients

\begin{tabular}{|c|c|c|}
\hline Adherence & Baseline values (\%) & Post-intervention (after counselling) (\%) \\
\hline High & $30(81.08)$ & $31(83.78)$ \\
\hline Medium & $6(16.21)$ & $6(16.21)$ \\
\hline Low & $1(2.70)$ & 0 \\
\hline
\end{tabular}


Table 8: Medication adherence-pre and post counselling in different stages of CKD

\begin{tabular}{|c|c|c|c|c|c|c|}
\hline \multirow{2}{*}{$\begin{array}{c}\text { CKD } \\
\text { Stage }\end{array}$} & \multicolumn{2}{|c|}{ High adhered } & \multicolumn{2}{c|}{ Medium adhered } & \multicolumn{2}{c|}{ Low adhered } \\
\cline { 2 - 6 } & Balues (\%) & $\begin{array}{c}\text { Post-intervention } \\
\text { (after } \\
\text { counselling) (\%) }\end{array}$ & $\begin{array}{c}\text { Baseline } \\
\text { values (\%) }\end{array}$ & $\begin{array}{c}\text { Post-intervention } \\
\text { (after counselling) } \\
\text { (\%) }\end{array}$ & $\begin{array}{c}\text { Baseline } \\
\text { values (\%) }\end{array}$ & $\begin{array}{c}\text { Post-intervention } \\
\text { (after } \\
\text { counselling) (\%) }\end{array}$ \\
\hline 3 to 5 & $49(74.2)$ & $52(78.8)$ & $12(18.2)$ & $10(15.15)$ & $2(3.03)$ & 0 \\
\hline 5D & $49(79)$ & $52(80.9)$ & $8(13)$ & $10(16.13)$ & $5(8.06)$ & 0 \\
\hline PT & $11(91.7)$ & $11(91.7)$ & $1(8.33)$ & $1(8.33)$ & 0 & 0 \\
\hline
\end{tabular}

Table 9: Age wise distribution of medication adherence-pre and post counselling

\begin{tabular}{|c|c|c|c|c|c|c|}
\hline \multirow[b]{3}{*}{ Age } & \multicolumn{6}{|c|}{ ADHERENCE } \\
\hline & \multicolumn{2}{|c|}{ High } & \multicolumn{2}{|c|}{ Medium } & \multicolumn{2}{|c|}{ Low } \\
\hline & $\begin{array}{c}\text { Baseline } \\
\text { values (\%) }\end{array}$ & $\begin{array}{l}\text { Post-intervention } \\
\text { (after } \\
\text { counselling) (\%) }\end{array}$ & $\begin{array}{c}\text { Baseline } \\
\text { values (\%) }\end{array}$ & $\begin{array}{l}\text { Post-intervention } \\
\text { (after } \\
\text { counselling) (\%) }\end{array}$ & $\begin{array}{c}\text { Baseline } \\
\text { values (\%) }\end{array}$ & $\begin{array}{l}\text { Post-intervention } \\
\text { (after } \\
\text { counselling) (\%) }\end{array}$ \\
\hline $20-29$ & $5(71.4)$ & $6(85.7)$ & $2(28.6)$ & $1(14.3)$ & 0 & 0 \\
\hline $30-39$ & $8(72.72)$ & $10(90.9)$ & $3(27.3)$ & $1(9.09)$ & 0 & 0 \\
\hline $40-49$ & $11(84.6)$ & $11(84.6)$ & $2(15.4)$ & $2(15.4)$ & 0 & 0 \\
\hline $50-59$ & $27(79.4)$ & $28(82.35)$ & 5 (14.7) & $6(17.64)$ & $2(5.88)$ & 0 \\
\hline $60-69$ & $36(78.26)$ & $41(89.13)$ & $6(13.04)$ & $5(10.87)$ & $4(8.7)$ & 0 \\
\hline $70-79$ & $20(80)$ & $20(80)$ & $2(8.0)$ & $5(16)$ & $2(8)$ & 0 \\
\hline $80-89$ & $3(75)$ & $3(75)$ & $1(25)$ & $1(25)$ & 0 & 0 \\
\hline
\end{tabular}

Table 10: Reasons for medication non-adherence-pre and post counselling

\begin{tabular}{|c|c|c|}
\hline Reasons & Baseline values (\%) & Post-intervention (after counselling) (\%) \\
\hline Forgetfulness & $14(10)$ & $05(3.57)$ \\
\hline Pill burden & $12(8.57)$ & $12(8.57)$ \\
\hline Concerned about cost & $12(8.57)$ & $12(8.57)$ \\
\hline Carelessness & $11(7.85)$ & $05(3.57)$ \\
\hline Fear of side effects & $07(5)$ & $01(0.71)$ \\
\hline Lack of information & $06(4.28)$ & 0 \\
\hline
\end{tabular}

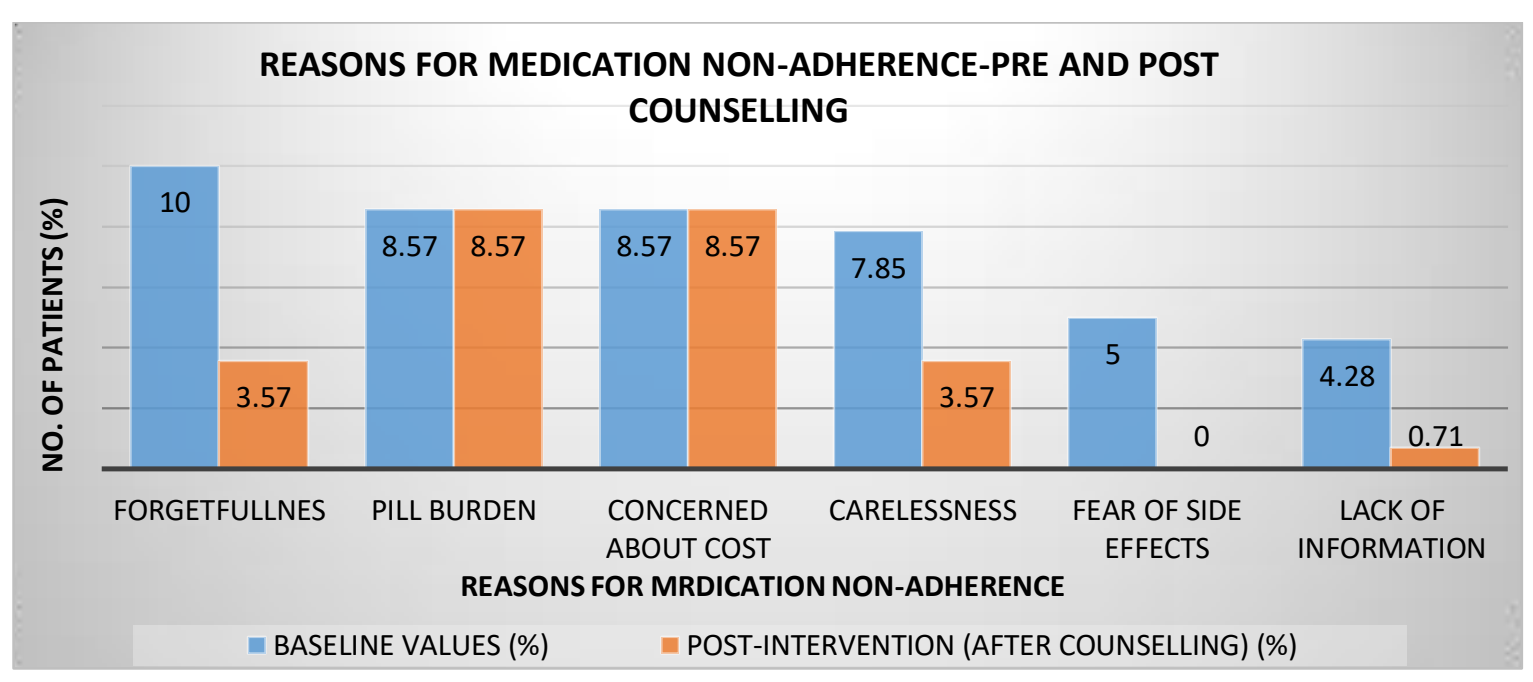

Figure 3: Graphical representation of reasons for medication non adherence-pre and post counselling 
Table 11: Number of pills taken by the patients

\begin{tabular}{|c|c|}
\hline Number of pills & Number of patients (\%) \\
\hline 1 to 5 & $21(15)$ \\
\hline 6 to 10 & $69(49)$ \\
\hline 11 to 15 & $44(32)$ \\
\hline$>15$ & $6(4)$ \\
\hline
\end{tabular}

Table 12: Quality of life of patients-pre and post counselling

\begin{tabular}{|l|c|c|c|}
\hline S. No. & Quality of life & $\begin{array}{c}\text { Mean value before } \\
\text { education }\end{array}$ & $\begin{array}{c}\text { Mean value after } \\
\text { education }\end{array}$ \\
\hline 1 & Emotional well being (EWB) & 8.4 & 8.46 \\
\hline 2 & Pain & 8.12 & 8.23 \\
\hline 3 & Social functioning (SF) & 7.30 & 7.43 \\
\hline 4 & Physical functioning (PF) & 6.49 & 6.51 \\
\hline 5 & Energy/fatigue (E/F) & 6.30 & 6.36 \\
\hline 6 & Role limitation to emotional problem & 6.25 & 5.98 \\
\hline 7 & (RLEP) & 5.94 & 6.07 \\
\hline 8 & Health change (HC) & 5.88 & 5.41 \\
\hline 9 & General health (GH) & 4.12 & 4.28 \\
\hline
\end{tabular}

Table 13: Quality of life in association with age and medication adherence-pre and post counselling

\begin{tabular}{|c|c|c|c|c|c|c|}
\hline \multirow{2}{*}{ Age (years) } & \multicolumn{2}{|c|}{ High adherence } & \multicolumn{2}{c|}{ Medium adherence } & \multicolumn{2}{c|}{ Low adherence } \\
\cline { 2 - 7 } & Baseline values & $\begin{array}{c}\text { Post- } \\
\text { intervention } \\
\text { (after } \\
\text { counselling) }\end{array}$ & Base line values & $\begin{array}{c}\text { Post- } \\
\text { intervention } \\
\text { (after } \\
\text { counselling) }\end{array}$ & $\begin{array}{c}\text { Base line } \\
\text { values }\end{array}$ & $\begin{array}{c}\text { Post-intervention } \\
\text { (after counselling) }\end{array}$ \\
\hline $20-29$ & 7.60 & 7.62 & 6.65 & 8.3 & - & - \\
\hline $30-39$ & 7.04 & 7 & 7.74 & 7.8 & - & - \\
\hline $40-49$ & 6.78 & 7.04 & 7.53 & 7.8 & - & - \\
\hline $50-59$ & 6.36 & 6.82 & 6.26 & 6.2 & 5.82 & - \\
\hline $60-69$ & 6.31 & 6.66 & 6.15 & 6.4 & 5.27 & - \\
\hline $70-79$ & 6.32 & 6.3 & 4.80 & 4.8 & 4.20 & - \\
\hline $80-89$ & 6.93 & 6.94 & 7 & 7 & & - \\
\hline
\end{tabular}

Table 14: Medication adherence before and after patient counselling

Paired Samples Correlations:

\begin{tabular}{|c|c|c|c|}
\hline & $\mathbf{N}$ & Correlation & Sig. \\
\hline Pair 1: $\quad$ Pre-adherence and post-adherence & 140 & 0.814 & 0.000 \\
\hline
\end{tabular}

Paired Sample Statistics:

\begin{tabular}{|c|c|c|c|c|c|}
\hline & & Mean & N & Std. Deviation & Std. Error Mean \\
\hline \multirow{2}{*}{ Pair 1 } & Pre-adherence & 0.44 & 140 & 0.969 & 0.082 \\
\cline { 2 - 6 } & Post-adherence & 0.26 & 140 & 0.653 & 0.055 \\
\hline
\end{tabular}

Paired sample test:

\begin{tabular}{|c|c|c|c|c|c|c|c|c|}
\hline \multirow{3}{*}{ Pair 1} & \multicolumn{5}{|c|}{$\begin{array}{l}\text { Paired differences } \\
\end{array}$} & \multirow{3}{*}{$\mathbf{T}$} & \multirow{3}{*}{ DF } & \multirow{3}{*}{$\begin{array}{l}\text { Sig. (2- } \\
\text { Tailed) }\end{array}$} \\
\hline & \multirow[t]{2}{*}{ Mean } & \multirow[t]{2}{*}{$\begin{array}{c}\text { Std. } \\
\text { Deviation }\end{array}$} & \multirow{2}{*}{$\begin{array}{c}\text { Std. } \\
\text { Error of } \\
\text { Mean }\end{array}$} & \multicolumn{2}{|c|}{$\begin{array}{l}95 \% \text { Confidence interval } \\
\text { of the difference }\end{array}$} & & & \\
\hline & & & & Lower & Upper & & & \\
\hline $\begin{array}{l}\text { Pre-adherence } \\
\text { and post- } \\
\text { adherence }\end{array}$ & 0.170 & 0.579 & 0.049 & 0.082 & 0.0275 & 3.652 & 139.00 & 0.000368048 \\
\hline
\end{tabular}




\section{DISCUSSION}

Chronic Kidney Disease (CKD), also known as chronic renal insufficiency, progressive kidney disease, or nephropathy, and it is defined as the presence of kidney damage or decreased Glomerular Filtration Rate (GFR) for 3months or more. CKD is a progressive disease that eventually leads to renal failure called End-Stage Renal diseases (ESRD). Therefore, effective management depends on making patient adhered to their medication. Good medication adherence is important to achieve desired therapeutic outcome whereas low adherence is considered as one of the major reason for decreased therapeutic outcome.

The aim was to evaluate medication adherence and to improve health related quality of life in patients with Chronic Kidney Disease. The study was done in NU Hospitals Bengaluru, Karnataka. The study was carried out for a period of 6 months from November 2018-April 2019, a total of 152 patients were included in the study. Out of 152 patients, 12 were excluded due to lack of follow up. Therefore, valuable data obtained was of 140 patients.

Our study included 140 patients as shown in Table 1, 103 were found to be male, 37 were found to be female and overall age group of patients range from 20-80 years of mean age $57.74 \pm 14.047$.

As shown in Table 2, in the age group of 20 to 29 years 7 $(5 \%)$, in the age group 30-39 years $11(7.86 \%)$, in the age group 40-49 years, 13 (9.3\%), in the age group $50-59$ years, $34(24.28 \%)$, in the age group $60-69$ years, $46(32.9 \%)$ in the age group $70-79$ years age group, $25(17.85 \%)$ and in the age group of 80 to $89,4(2.85 \%)$ patients were found. Highest percentage of patients was found in the age group of 50-70 years.

In a study conducted by Syeda Firdous, Ayesh Siddiqua et.al. on "Assessment of risk factors and medication adherence of CKD", it was found that 41-50 years of age group patients were more adhered i.e. $92(30 . \%)$ as compared to remaining age groups 4 .

In current study as shown in Table 3 , it was found that $15.7 \%$ belong to CKD stage $3,17.85 \%$ belong to CKD stage 4 , $13.57 \%$ belong to CKD stage $5,44.28 \%$ were on haemodialysis and $8.57 \%$ were CKD transplant patients.

Siva Kala T, Arepalli Sreedevi, Hari Prasad MV, Jikki PN conducted a study on "Assessment of knowledge and adherence to therapy among CKD patients". It was observed that $68.9 \%$ were in stage $5,15.53 \%$ stage $4,11 \%$ were stage 3 among which $85 \%$ patients were found to be on $\mathrm{HD}^{6}$.

Out of 140 patients included in our study, as shown in Table 4 it was found that the comorbidity associated with CKD was HTN (94.2\%), DM (61.4\%), anemia (37.1\%), cardiovascular disease $(20 \%)$, thyroid $(15 \%)$ and respiratory disorder $(7.14 \%)$. In our study, it was found that 132 out of 140 patients had HTN (94.2\%) than any other co-morbidity.

In a previous study by Olumuyiwa J.F, Peter Ehizokhale Akhideno, Oluwatosin Beatrice Ibiyemi-Fasipe et. al. on "The burden of polypharmacy and pattern of comorbidities among chronic kidney disease patients in clinical practice" it was observed that the main co-morbidity associated with CKD were HTN (83.70\%), DM (31.70\%), Coronary Heart Disease (8.90\%) and obesity (19.51\%) ${ }^{7}$.

Out of 140 patients, as shown in Table 5, high adherence was found in 112 (80\%), medium adherence was found in 21 $(45 \%)$ and low adherence was found in 7 (5\%) prior to education. For patients whose medication adherence was low during the baseline visit which was improved by patient education through counselling. Patient with low and medium adherence during the first visit were 7 (5\%) and 21 $(45 \%)$ which were gradually reduced and patients with high adherence during baseline visit were $80 \%$, which later improved to $85.7 \%$ in the final review.

In a study conducted by W. Tesfaye, R. Castelino et.al. on "Medication adherence in adults with advanced Chronic Kidney Disease", it was observed that 35 patients out of 78 were found to be non adhered ( $45 \%$ ) and $55 \%$ patients were found to be adhered8.

In this study as shown in Table 6and 7, it was found that small range of significant difference exist between medication adherence in male and female i.e., females were found to be more adhered to medication by $81.08 \%$ than male $79.61 \%$.

A similar study conducted by Yoke Mun Chan, Mohd Shariff et.al. on "Determinants of compliance behaviour among patients undergoing haemodialysis", it was found that there were no significant difference between male and female subjects on medication compliance ${ }^{9}$.

Out of 140 patients included in our study as shown in Table 8, CKD transplant patients were found to be more adhered than patients undergoing dialysis and patients of CKD stages 3-5.

In a study conducted by Rajiv Ahlawat, Pramil Tiwari and Sanjay D Cruz, on "Prevalence and predictors of medication non adherence in patients of CKD", it was found that patients on haemodialysis were more likely to be non-adherent to medications compared to other stages of CKD ${ }^{10}$.

In our study it was found that as shown in Table 9, younger adults of age group 20-29 years were less adhered to their medication regimen compared to older age and also medication adherence was improved after effective patient education through counselling.

In a previous study conducted by Tangkiatkumjai M, Walker $\mathrm{D} \mathrm{M}$ et. al. on "Association between medication adherence and clinical outcomes in patients with CKD". It was found that patients in the younger age group 28-39 years reported medication non-adherence at higher rate $(38 \%)$ than patients over 60 years $(21 \%)^{11}$.

In present study, as shown in Table 10, it was found that the most common reason for non-adherence was forgetfulness (10\%), carelessness (7.85), concern about cost $(8.57 \%)$, pill burden $(8.57 \%)$, fear of side effect $(5 \%)$ and the least effected factor was lack of information (4.28\%). After patient education, it was found that forgetfulness reduced to $3.57 \%$, carelessness reduced to $3.57 \%$, fear of side effects was 0 and lack of information reduced to $0.71 \%$. But there was no change found in pill burden and concern in cost.

In a study conducted by Bhupendra Verma, Amrita Singh, J. S. Bishnoi, Anil Kumar Mishra conducted a study on "Adherence to medication in chronic kidney disease". It was found that High cost of medications (72\%) and need to take medicines for long durations (59\%) were among the most common types of non-adherence. High pill burden and complex dosing schedule were other common causes of nonadherence in this study. Prescription of $\geq 4-5$ pills/day has been significantly associated with non-adherence in CKD patients. Fear of adverse effects of medications, lack of knowledge about necessity of individual drug, and lack of knowledge and insight about nature of disease were other important factors ${ }^{12}$.

In our study, as shown in Table 11, out of 140 patients, it was found that 21 patients had a daily pill load of 1 to 5,69 
patients had a pill load of 6-10, 4 patients had a pill load of $11-15$ and 6 patients had $>15$ pill load.

In a study conducted by Yi-Wen Chiu, Isaac Teitelbaum, Madhukar Misra on "Pill burden, adherence, hyperphosphatemia, and quality of life in maintenance dialysis patients". It was observed that the oral medications contributed to a median daily pill burden of 19 . The daily pill burden exceeded 10 in $91 \%$ of subjects, was more than 20 in $47 \%$, and more than 30 in $17 \% 13$.

Quality of Life was assessed for 140 patients before and after patient counselling. It was found that as shown in Table 12 after patient counselling there was a slight improvement in Quality of Life of the patients with CKD. The domain with highest average score was Emotional well being (8.4) and lowest average score was role limitation due to physical health (4.12).

In a study conducted by Keila Batista Alves, Nathalia and Cristina Sanches, on "Is quality of life associated with compliance to pharmacotherapy in patients with chronic kidney disease undergoing maintenance hemodialysis" it was found that the domain with lowest average score were physical role functioning $(0.0)$ and emotional role functioning $(33.3 \%)$. On the other hand, the domain social role functioning showed best average score $(100.0 \%)^{5}$.

In our study it was found that as shown in Table 13, younger adults of age group 20-29 years were having better QOL compared to older age.

Correlation between medication adherence and quality of life was done using Pearson correlation. Although the quality of life score had slight association with medication adherence, the change was not statistically significant because the mean difference was found to be low.

Patient's adherence to medication was assessed before and after patient counselling using paired Sample-T test as shown in Table 14. It was found that patients adherence to medication was improved after patient education through patient counselling. Out of 140 patients, prior to patient education during baseline visits forgetfulness was found in 14 patients, which was reduced to 5 patients, carelessness was found in 11 patients which was reduced to 5 patients, fear of side effects was found in 7 patients which was reduced to 0 patients and lack of information was found in 6 patients which was reduced to 1 patient after patient education through counselling. Thus, the study was found to be statistically significant ( $\mathrm{p}$ value $<0.05$ ).

In a study conducted by Anurodh Ghimirey, Binaya Sapkota, Sweta Shrestha on "Evaluation of pharmacist counselling in improving knowledge, attitude, and practice in chronic kidney disease patients"; it was observed that mean knowledge score of the case group after intervention was significantly higher than that before intervention. The knowledge score increased from $8.06 \pm 4.25$ to $8.22 \pm 4.13$ in patients, the change was not statistically significant ${ }^{14}$.

\section{CONCLUSION}

Medication adherence is a dynamic behaviour and need constant monitoring. Non adherence to medication is very prevalent among CKD patients in India, which further increases with duration of treatment. Low medication adherence was significantly associated with mortality and disease progression. Non compliance to drug therapy also worsens the quality of life.

It was found that patients adherence to medication was improved from 80 to $85 \%$ after patient counselling, thus this study demonstrates that patients adherence to medication can be improved by educating the patient through counselling.

We found that forgetfulness; carelessness and concern about cost were the major reasons contributing for medication non adherence. HTN was the most common co-morbidity among CKD patients.

An adequate education through patient counselling was carried out to the patient to improve their medication adherence and to make them understand the importance of being adhered to the medication. The domain with highest average score was emotional well being and lowest average score was role limitation due to physical health. Patient's quality of life was slightly improved after patient counselling.

From this study we analysed the reasons contributing for medication non adherence and with the help of patient counselling we were able to improve medication adherence and improve quality of life in patients with CKD.

\section{Limitations}

KDQOL assessment was tedious and time consuming due to communication barriers and patient compliance.

\section{Future Directions}

$>$ If the study is multicentered, more reproducible data can be obtained for a larger population.

$>$ If the study is carried out for a longer period of time more reliable data can be obtained.

\section{ACKNOWLEDGEMENT}

The authors wish to thank the management of PES College of Pharmacy, Bengaluru, Karnataka, India for providing necessary equipment for research, constant encouragement, facilities and support.

\section{CONFLICTS OF INTEREST}

\section{The author declares that there is no conflict of interest to disclose.}

\section{REFERENCES}

1. Saran R, Robinson B, Abbott KC, Agodoa LY, Bhave N, Bragg-Gresham J, Balkrishnan R, Dietrich X, Eckard A, Eggers PW, Gaipov A. US renal data system 2017 annual data report: epidemiology of kidney disease in the United States. American Journal of Kidney Diseases 2018; 71(3):A7.

2. Dasari P, Venkateshwarlu K, Venisetty R. Management of comorbidities in chronic kidney disease: a prospective observational study. Int J Pharm Pharm Sci 2014; 6(2):363-7.

3. Kramer H, Berns JS, Nally J, Choi MJ, Rocco MV. A decade after the KDOQI CKD guidelines: impact on NKF-KDOQI. American Journal of Kidney Diseases 2012; 60(5):694-6.

4. Firdous SS, Siddiqua A, Navaneetha K, Srujana P. Assessment of risk factors and medication adherence of chronic kidney disease patients in a tertiary care teaching hospital 2014; 2:6.

5. Alves KB, Guilarducci NV, Santos TD, Baldoni AO, Otoni A, Pinto SW, Zanette C, Sanches C. Is quality of life associated with compliance to pharmacotherapy in patients with chronic kidney disease undergoing maintenance hemodialysis? 2018; 16(1).

6. Siva Kala T, Arepalli Sreedevi, Hari Prasad MV, Jikki PN. Assessment of knowledge and adherence to therapy among chronic kidney disease patients attending nephrology department of tertiary care hospital, Kurnool city, Andhra Pradesh. International Journal of Medical Science and Public Health 2019; 8(3):223-9. 
7. Fasipe OJ, Akhideno PE, Ibiyemi-Fasipe OB, Idowu AA. The burden of polypharmacy and pattern of comorbidities among chronic kidney disease patients in clinical practice. Archives of Medicine and Health Sciences 2018; 6(1):40.

8. Tesfaye W, Castelino RL, Wimmer BC, Mckercher C, Jose MD, Peterson GM, Zaidi ST. Medication adherence in adults with advanced chronic kidney disease. In nephrology 2018; 25:44-45.

9. Chan YM, Zalilah MS, Hii SZ. Determinants of compliance behaviours among patients undergoing hemodialysis in Malaysia. PLoS One 2012; 7(8):e41362.

10. Ahlawat R, Tiwari P, D'Cruz S. Prevalence and predictors of medication non-adherence in patients of chronic kidney disease: Evidence from a cross sectional study. J Pharma Care Health Sys 2016; 3(152):2376-419.

11. Tangkiatkumjai $M$, Walker DM, Praditpornsilpa $K$, Boardman H. Association between medication adherence and clinical outcomes in patients with chronic kidney disease: a prospective cohort study. Clinical and experimental nephrology 2017; 21(3):504-12.

12. Verma B, Singh A, Bishnoi JS, Mishra AK. Adherence to Medications in Chronic Kidney Disease: Prevalence, Predictors and Outcomes. Int J Cur Res Rev 2018; 10(19):14.

13. Chiu YW, Teitelbaum I, Misra M, De Leon EM, Adzize T, Mehrotra R. Pill burden, adherence, hyperphosphatemia, and quality of life in maintenance dialysis patients. Clinical Journal of the American Society of Nephrology 2009; 4(6):1089-96.

14. Ghimirey A, Sapkota B, Shrestha S, Basnet N, Shankar PR, Sapkota S. Evaluation of pharmacist counseling in improving knowledge, attitude, and practice in chronic kidney disease patients. SAGE open medicine 2013; 1(12):1-5. 